\title{
Genetic Diversity within the Genus Pleurotus Determined by RAPD Analysis
}

\author{
Ravi Prakash Mishra, Manjul Pandey*, U.K. Tripathi and M. Singh \\ Department of Plant Pathology, C.S. Azad University of Agriculture and Technology, \\ Kanpur-208002, India \\ *Corresponding author
}

Keywords

Genomic DNA polymorphism, RAPD, Pleurotus species, Clusters

Article Info

Accepted:

17 December 2018

Available Online:

10 January 2019

\section{A B S T R A C T}

Random amplified polymorphism DNA (RAPD) analysis was done to assess the diversity among ten species of Pleurotus. Understanding the pattern of fundamental not only addressing questions concerning evolutionary process and the development of conservation strategies, but also a prerequisite of the efficient use of genetic resources in breeding programme. The RAPD dendogram obtained by using a UPGMA programme grouped in to the investigated strains in to 5 clusters. RAPD bands were scored as present (1) or absent (0) for all the Pleurotus isolate. Each band was assumed to represent a unique genetic locus. The pattern and extent of RAPD variation were analyzed with respect to primer, polymorphic locus and isolate. Total number of amplified fragment and polymorphic fragment produced by 40 decamer primer were 229 and 226, respectively. Polymorphism percentage was 98.69. Ten primer SBSA11, SBSA13, SBSA15, SBSA16, SBSA18, SBSA19, SBSA20, SBSB14, SBSB15 and SBSB17 were not amplified the DNA from any of the isolate.

\section{Introduction}

Apart from the culinary, nutritional and health benefits of edible mushrooms, its large scale cultivation is now playing an instrumental role in solving one of the main problems facing mankind in the $21^{\text {th }}$ century; the need to feed an ever-increasing population (Gokulpalan and Nair, 1990). Mushrooms have been recognized by Food and Agriculture Organization as food contributing to ameliorate the protein malnutrition in human body in the industrial waste materials; are rich in the protein, minerals and vitamins; and contain an abundance of the essential aminoacid lysine (Thayumanavan and Manikam, 1980). Therefore, mushrooms can be a good supplement to cereals. Development countries like ours need nutrients substitutes into the staple diet of man will essentially increase proteins for human consumption.

The genus Pleurotus is a heterogeneous group of several species are having nutritional and medicinal importance (Gunde-Cimerman, 1999 and Guzman, 2000). Some Pleurotus spp. Have the ability to absorb microelements from the different cultivation media, and thus 
they may present an excellent dietary source (Stajic et al., 2002). The interest in the genetic structure of natural population has increased in the last few years owning to the necessity to broaden the knowledge of genetic variation in cultivated species. New approach to the study of genetic variation from wild species to cultivated varieties, mediated by information on molecular markers is promising avenues to exploit wild genetic resource in breeding programme. In fact, despite the economic importance of species in general or fungi in particular, little was known until recently about their natural population and the available genetic variability (Zervakis et al.,2004).An attempt was made in the present study to find out the genetic variability present in Pleurotus species by RAPD method.

\section{Materials and Methods}

\section{DNA isolation}

Pleurotus species under study were grown at room temperature on PDA for 15 to 18 days. Mycelium was collected by scrapping. The harvested mycelium (100 mg) was placed in pre-cooled mortar and pestle and liquid nitrogen was added. The mycelium was powdered then this was transferred into an Eppendrop $(1.5 \mathrm{ml})$ tube containing $1 \mathrm{ml}$ CTAB buffer and placed in heating block at $65^{\circ} \mathrm{C}$ for 15 minutes. During incubation period, mixed by hand 2 to 3 times and centrifuged it at $13000 \mathrm{rpm}$ for 5 minute. Supernatant was taken out into a fresh Eppendrop tube and $1 \mathrm{ml} \mathrm{GN}$ binding buffer was added, mixed by inversion. Then it was transferred to a mini prep spin column with a $2 \mathrm{ml}$ collection tube. Let it kept for 3 minutes. Centrifuged at 13000, rpm for 30 seconds and the flow through was discarded. The rest of the solution was added in to the column and centrifuged for 30 seconds at $13000 \mathrm{rpm}$. Washing buffer $(600 \mu \mathrm{l})$ was added and centrifuged for 30 seconds a same rpm, this step was repeated for one more time to remove impurities as much as possible then centrifuged at $13000 \mathrm{rpm}$ for an additional 1 minute to remove the residual washing buffer. Spin column was placed into a $1.5 \mathrm{ml}$ micro tube. $\mathrm{T}_{10} \mathrm{E}_{1}$ buffer $(100 \mu \mathrm{l})$ was added into the center part of the Si Max membrane in the spin column and incubated at room temperature for 3 to 5 minutes and centrifuged at $13000 \mathrm{rpm}$ for 1 minute to elute DNA. The quality of the purified was determined on 1 per cent agarose gel stained with Goldview ${ }^{\mathrm{Tm}}$ or ethidium bromide. The purified DNA was stored at $4^{0} \mathrm{C}$ for further use.

\section{RAPD primer}

Random primers were procured from SBS Genetech company Ltd. the list of the primers name and sequences is listed in Table 1.

\section{PCR-Mix}

The reaction mixture of $25 \mu 1$, each containing primer $-2 \mu \mathrm{l}\left(50 \mathrm{pm} \mathrm{ml}^{-1}\right)$, dNTP mix $-2 \mu \mathrm{l}$, $\mathrm{MgCl}_{2}-1 \mu \mathrm{l}$, Taq DNA polymerase $-1 \mu \mathrm{l}(5 \mathrm{U}$ $\mathrm{ml}^{-1}$ Genetech.), 10 X PCR buffer $-2.5 \mu \mathrm{l}$ (100 $\mathrm{mM}$ Tris HCL (pH 8.3), and $13.5 \mathrm{ml}$ of $\mathrm{H}_{2} \mathrm{O}$. To this $4 \mu 1$ genomic DNA was added.

\section{Thermal cycler condition}

Amplification was performed in a master cycler with lid heating option at $105^{\circ} \mathrm{C}$ with initial denaturation of genomic DNA at $95^{\circ} \mathrm{C}$ for $2 \mathrm{~min}$. followed by 35 cycles of template denaturation at $94^{\circ} \mathrm{C}$ for $1 \mathrm{~min}$. primer annealing at $36^{\circ} \mathrm{C}$ for $45 \mathrm{sec}$, extension at $72^{\circ} \mathrm{C}$ for $2 \mathrm{~min}$ and a final extension at $72^{\circ} \mathrm{C}$ for $10 \mathrm{~min}$.

\section{Gel electrophoresis}

A $200 \mathrm{ml}$ (1XTAE) agarose gel was prepared. For making Gel, $180 \mathrm{ml}$ distilled water +20 $\mathrm{ml}$ TAE buffer $(10 \mathrm{X})+$ agarose $2 \mathrm{~g}$ was 
added and boiled to dissolved, and then kept it for cooling. The comb in gel casting tray was fixed and agarose solution was poured slowly. It was kept for 30 minutes to solidify the gel. The comb was pulled out. $1 \mathrm{~kb}$ DNA ladder (2 $\mu 1+2 \mu \mathrm{l}$ TAE $)+2 \mu 16 \mathrm{X}$ loading dye was loaded in first well, in subsequent wells the PCR amplified product $(5 \mu 1)$ was loaded. The gel was run in $1 \mathrm{X}$ TAE buffer at $60 \mathrm{~V}$ for 2 hrs. The gel was stained with ethidium bromide for 20 minute.

\section{Visualization of gel}

The gel was visualized with gel documentation system (UVI Tek, UK) and the photograph was taken.

\section{Statistical data analysis}

RAPD bands were scored as present (1) or absent (o) for all the Pleurotus isolates. Each band was assumed to represent a unique genetic locus. Its presence was interpreted as either a heterozygote or dominant homozygote and absence of the band as a recessive homozygote. The pattern and extent of RAPD variation were analyzed with respect to primer, polymorphic locus and isolate. Data entry was done into binary data matrix and statistical analysis was carried out using NTSYS-PC, 2.01 version (Rohlf, 1997). Pair wise comparison of samples was used to estimate Jaccards similarity coefficient. Genetic distances (GD) between pair of lines were estimated at 1-GS. Jaccard's similarity coefficient was used to generate dendogram using unweighted pair group method with arithmetic mean UPGMA (Sneath and Sokal, 1973).

\section{Results and Discussion}

All Pleurotus species included in this study showed unique banding pattern as observed from amplification band comparisons obtained used by 40 decamer primers. Total number of amplified fragments and polymorphic fragments produced by 40 decamer primers were 229 and 226, respectively.

Table.1 The list of the primers name and sequences used in this study

\begin{tabular}{|l|l|l|l|l|}
\hline 1. & SBSA-01 & CAG GCC CTT C & SBSB-01 & GTT TCG CTC C \\
\hline 2. & SBSA-02 & TGC CGA GCT G & SBSB-02 & TGA TCC CTG G \\
\hline 3. & SBSA-03 & AGT CAG CCA C & SBSB-03 & CAT CCC CCT G \\
\hline 4. & SBSA-04 & AAT CGG GCT G & SBSB-04 & GGA CTG GAG T \\
\hline 5. & SBSA-05 & AGG GGT CTT G & SBSB-05 & TGC GCC CTT C \\
\hline 6. & SBSA-06 & GGT CCC TGA C & SBSB-06 & TGC TCT GCC C \\
\hline 7. & SBSA-07 & GAA ACG GGT G & SBSB-07 & GGT GAC GCA G \\
\hline 8. & SBSA-08 & GTG ACG TAG G & SBSB-08 & TGC CAC ACG G \\
\hline 9. & SBSA-09 & GGG TAA CGC C & SBSB-09 & TGG GGG ACT C \\
\hline 10. & SBSA-10 & GTG ATC GCA G & SBSB-10 & CTG CTG GGA C \\
\hline 11. & SBSA-11 & CAA TCG CCG T & SBSB-11 & GTA GAC CCG T \\
\hline 12. & SBSA-12 & TCG GCG ATA G & SBSB-12 & CCT TGA CGC A \\
\hline 13. & SBSA-13 & CAG CAC CCA C & SBSB-13 & TTC CCC CGC T \\
\hline 14. & SBSA-14 & TCT GTG CTG G & SBSB-14 & TCC GCT CTG G \\
\hline 15. & SBSA-15 & TTC CGA ACC C & SBSB-15 & GGA GGG TGT T \\
\hline 16. & SBSA-16 & AGC CAG CGA A & SBSB-16 & TTT GCC CGG A \\
\hline 17. & SBSA-17 & GAC CGC TTG T & SBSB-17 & AGG GAA CGA G \\
\hline 18. & SBSA-18 & AGG TGA CCG TA & SBSB-18 & CCA CAG CAG T \\
\hline 19. & SBSA-19 & CAA ACG TCG G & SBSB-19 & ACC CCC GAA G \\
\hline 20. & SBSA-20 & GTT GCG TCG G & SBSB-20 & GGA CCC TTA C \\
\hline
\end{tabular}


Table.2 List of decamer primer and the polymorphism observed in the study

\begin{tabular}{|c|c|c|c|c|c|c|}
\hline $\begin{array}{l}\text { Sl. } \\
\text { No. }\end{array}$ & Primer & Primer Sequence & $\begin{array}{l}\text { Total no. } \\
\text { of bands }\end{array}$ & $\begin{array}{l}\text { No.of } \\
\text { Polymorphic } \\
\text { bands }\end{array}$ & $\begin{array}{l}\text { Polymor } \\
\text { phism } \\
\text { Per cent }\end{array}$ & Isolate Distinguished \\
\hline 1. & SBSA-01 & CAG GCC CTT C & 7 & 7 & 100 & $\mathrm{P} 1(325,500 \mathrm{bp})$ \\
\hline 2. & SBSA-02 & TGC CGA GCT G & 8 & 8 & 100 & $\mathrm{P} 5(225,675 \mathrm{bp})$ and $\mathrm{P} 8(300 \mathrm{bp})$ \\
\hline 3. & SBSA-03 & AGT CAG CCA C & 9 & 9 & 100 & P6(350 bp) \\
\hline 4. & SBSA-04 & AAT CGG GCT G & 8 & 6 & 75 & P8(400 bp) \\
\hline 5. & SBSA-05 & AGG GGT CTT G & 9 & 9 & 100 & P6(575 bp) \\
\hline 6. & SBSA-06 & GGT CCC TGA C & 8 & 8 & 100 & $\mathrm{P} 6(740 \mathrm{bp})$ and $\mathrm{P} 8(500,625 \mathrm{bp})$ \\
\hline 7. & SBSA-07 & GAA ACG GGT G & 10 & 10 & 100 & $\mathrm{P} 2(450 \mathrm{bp})$ and $\mathrm{P} 8(750 \mathrm{bp})$ \\
\hline 8. & SBSA-08 & GTG ACG TAG G & 8 & 8 & 100 & $\mathrm{P} 6(500,800 \mathrm{bp}) ; \mathrm{P} 9(650 \mathrm{bp})$ and $\mathrm{P} 10(375 \mathrm{bp})$ \\
\hline 9. & SBSA-09 & GGG TAA CGC C & 5 & 5 & 100 & $\begin{array}{l}\mathrm{P} 1(450,775 \mathrm{bp}) ; \mathrm{P} 6(730,925 \mathrm{bp}) \text { and } \mathrm{P} 8(425 \\
\text { bp) }\end{array}$ \\
\hline 10. & SBSA-10 & GTG ATC GCA G & 8 & 8 & 100 & P8(475 bp) \\
\hline 11. & SBSA-11 & CAA TCG CCG T & - & - & - & - \\
\hline 12. & SBSA-12 & TCG GCG ATA G & 10 & 10 & 100 & $\mathrm{P} 1(690 \mathrm{bp}) ; \mathrm{P} 7(375,650 \mathrm{bp})$ and $\mathrm{P} 9(250,675 \mathrm{bp})$ \\
\hline 13. & SBSA-13 & CAG CAC CCA C & - & - & - & - \\
\hline 14. & SBSA-14 & TCT GTG CTG G & 7 & 6 & 85.7 & $\mathrm{P} 10(525,600 \mathrm{bp})$ \\
\hline 15. & SBSA-15 & TTC CGA ACC C & - & - & - & - \\
\hline 16. & SBSA-16 & AGC CAG CGA A & - & - & - & - \\
\hline 17. & SBSA-17 & GAC CGC TTG T & 8 & 8 & 100 & $\begin{array}{l}\mathrm{P} 4(300,525,600 \mathrm{bp}) ; \mathrm{P} 8(850 \mathrm{bp}) \text { and } \\
\mathrm{P} 9(650,1000 \mathrm{bp})\end{array}$ \\
\hline 18. & SBSA-18 & AGG TGA CCG T & - & - & - & - \\
\hline 19. & SBSA-19 & CAA ACG TCG G & - & - & - & - \\
\hline 20. & SBSA-20 & GTT GCG TCG G & - & - & - & - \\
\hline 21. & SBSB-01 & GTT TCG CTC C & 6 & 6 & 100 & P6(750 bp) ; P9(250 bp) \\
\hline 22. & SBSB-02 & TGA TCC CTG G & 8 & 8 & 100 & $\mathrm{P} 1(490 \mathrm{bp}) ; \mathrm{P} 6(1000 \mathrm{bp})$ \\
\hline 23. & SBSB-03 & CAT CCC CCT G & 5 & 5 & 100 & - \\
\hline 24. & SBSB-04 & GGA CTG GAG T & 8 & 8 & 100 & $\mathrm{P} 10(625 \mathrm{bp})$ \\
\hline 25. & SBSB-05 & TGC GCC CTT C & 7 & 7 & 100 & P8(675 bp) \\
\hline 26. & SBSB-06 & TGC TCT GCC C & 9 & 9 & 100 & P6(375 bp);P8(650 bp) \\
\hline 27. & SBSB-07 & GGT GAC GCA G & 6 & 6 & 100 & - \\
\hline 28. & SBSB-08 & TGC CAC ACG G & 9 & 8 & 88.8 & P6(750 bp); P8(625 bp) \\
\hline 29. & SBSB-09 & TGG GGG ACT C & 7 & 7 & 100 & $\mathrm{P} 2(300 \mathrm{bp})$ and $\mathrm{P} 9(750 \mathrm{bp})$ \\
\hline 30. & SBSB-10 & CTG CTG GGA C & 7 & 7 & 100 & P19275 bp); P8(250bp) and P9,P10(625 bp) \\
\hline 31. & SBSB-11 & GTA GAC CCG T & 8 & 8 & 100 & $\mathrm{P} 9(375 \mathrm{bp})$ \\
\hline 32. & SBSB-12 & CCT TGA CGC A & 8 & 8 & 100 & P3(750 bp); P8(300,990 bp); P9(550 bp) \\
\hline 33. & SBSB-13 & TTC CCC CGC T & 7 & 7 & 100 & $\mathrm{P} 9(900 \mathrm{bp})$ and $\mathrm{P} 10(450 \mathrm{bp})$ \\
\hline 34. & SBSB-14 & TCC GCT CTG G & - & - & - & - \\
\hline 35. & SBSB-15 & GGA GGG TGT T & - & - & - & - \\
\hline 36. & SBSB-16 & TTT GCC CGG A & 4 & 4 & 100 & $\mathrm{P} 1(675 \mathrm{bp})$ \\
\hline 37. & SBSB-17 & AGG GAA CGA G & - & - & - & - \\
\hline 38. & SBSB-18 & CCA CAG CAG T & 8 & 8 & 100 & $\mathrm{P} 9(350,500 \mathrm{bp})$ \\
\hline 39. & SBSB-19 & ACC CCC GAA G & 9 & 9 & 100 & $\mathrm{P} 8(350 \mathrm{bp})$ and $\mathrm{P} 9(400 \mathrm{bp})$ \\
\hline \multirow[t]{2}{*}{40.} & SBSB-20 & GGA CCC TTA C & 10 & 9 & 90 & $\mathrm{P} 10(375 \mathrm{bp})$ \\
\hline & Total & & 229 & 226 & 98.69 & \\
\hline
\end{tabular}


Table.3 Genetic similarity co-efficient based on 40 RAPD primers among 10 species of Pleurotus

\begin{tabular}{|c|c|c|c|c|c|c|c|c|c|c|c|}
\hline $\begin{array}{l}\text { Sl. } \\
\text { No. }\end{array}$ & Species & $\begin{array}{c}P . \\
\text { sajor } \\
\text { caju }\end{array}$ & $\begin{array}{c}P . \\
\text { flabellat } \\
\text { us }\end{array}$ & $\begin{array}{c}P . \\
\text { platypus }\end{array}$ & $\begin{array}{c}P . \\
\text { fossulatu } \\
s\end{array}$ & $\begin{array}{c}P . \\
\text { florida }\end{array}$ & $\begin{array}{c}P . \\
\text { citrinopil } \\
\text { eatus }\end{array}$ & $\begin{array}{c}P . \\
\text { sapid } \\
\text { us }\end{array}$ & $\begin{array}{c}P . \\
\text { dajmor }\end{array}$ & $\begin{array}{c}P . \\
\text { ostreatu } \\
S\end{array}$ & $\begin{array}{c}\text { H. } \\
\text { ulmarious }\end{array}$ \\
\hline 1 & P. sajor caju & 1.000 & & & & & & & & & \\
\hline 2 & P. flabellatus & 0.198 & 1.000 & & & & & & & & \\
\hline 3 & P. platypus & 0.191 & 0.527 & 1.000 & & & & & & & \\
\hline 4 & P. fossulatus & 0.198 & 0.509 & 0.568 & 1.000 & & & & & & \\
\hline 5 & P. florida & 0.200 & 0.513 & 0.574 & 0.988 & 1.000 & & & & & \\
\hline 6 & P.citrinopileatus & 0.333 & 0.122 & 0.169 & 0.150 & 0.151 & 1.000 & & & & \\
\hline 7 & P.sapidus & 0.204 & 0.500 & 0.840 & 0.581 & 0.5871 & 0.174 & 1.000 & & & \\
\hline 8 & P. dajmor & 0.177 & 0.221 & 0.203 & 0.183 & 0.184 & 0.133 & 0.189 & 1.000 & & \\
\hline 9 & P.ostreatus & 0.152 & 0.350 & 0.314 & 0.263 & 0.265 & 0.192 & 0.317 & 0.188 & 1.000 & \\
\hline 10 & H. ulmarious & 0.166 & 0.401 & 0.313 & 0.344 & 0.347 & 0.141 & 0.305 & 0.241 & 0.447 & 1.000 \\
\hline
\end{tabular}

Fig.1 UPGMA dendogram depicting relationship among ten Pleurotus species

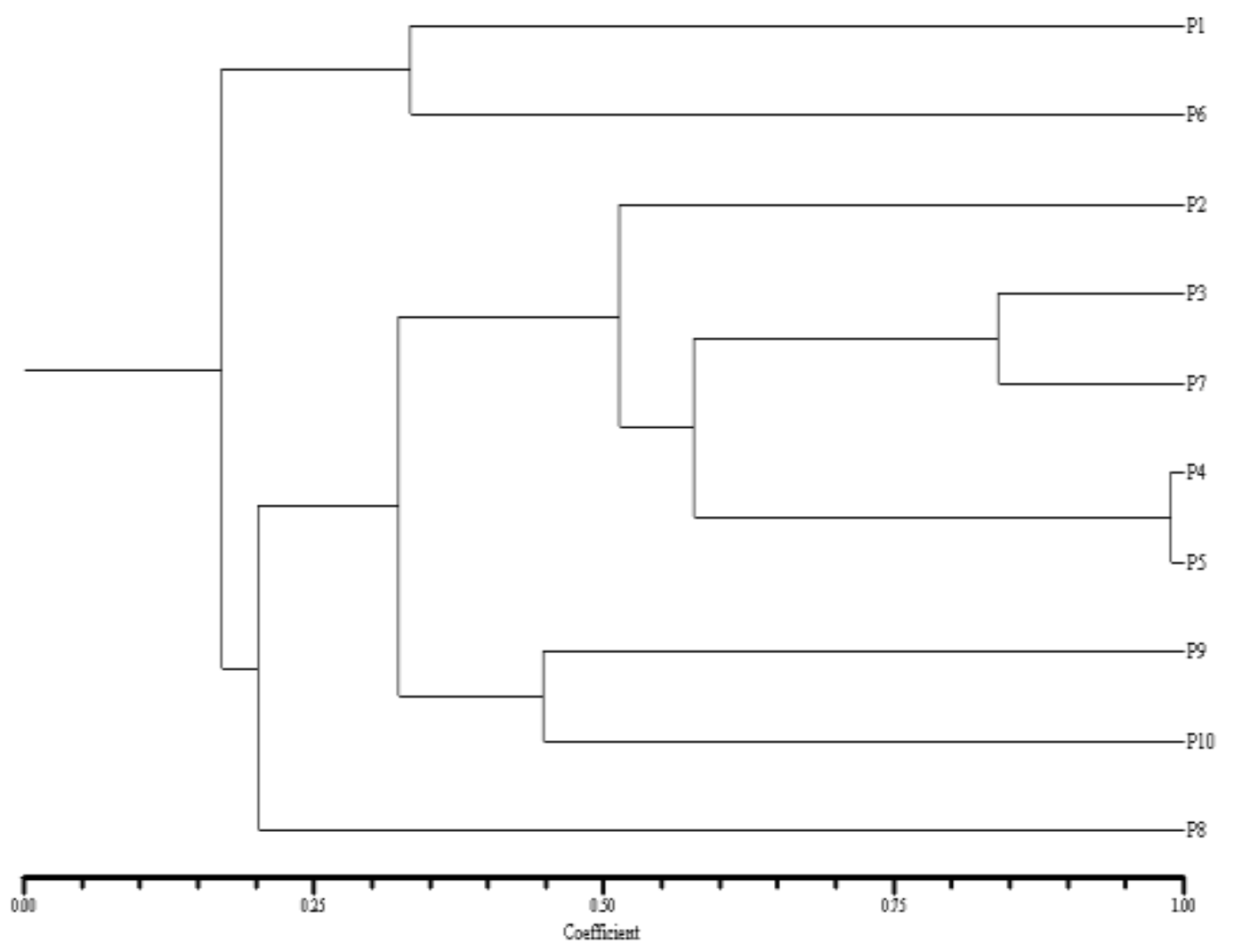

Polymorphism percentage was 98.69. Ten primers namely SBSA 11, SBSA 13, SBSA 15, SBSA 16, SBSA 18, SBSA 19, SBSA 20, SBSB 14, SBSB 15, SBSB 17 were not amplified the DNA from any of the isolate. Some of the RAPD primers were found to be specific to distinguished the Pleurotus species. Primer SBSA 01 would distinguished P. sajor caju (325 bp, 500 bp). Primer SBSA $07 \&$ SBSB 09 would distinguished $P$. flabellatus (450bp \& 300bp respectively). $P$. platypus be distinguished by primer SBSB 12 
(700bp), primer SBSA 17 will be identified the strain P. fossulatus $(300 \mathrm{bp}, 525 \mathrm{bp}, 600$ $\mathrm{bp}$ ), primer SBSA 02 would distinguished sp. P. florida (225 bp, 375 bp, 875 bp), SBSA 05, SBSA 08, SBSB 06 will distinguish $P$. citrinopileatus (575 bp, 500 bp \& 800 bp, 375 bp respectively), SBSA 12 will be able to identify $P$. sapidus (375 bp, 650 bp), $P$. djamor will be distinguished by SBSB $19 \&$ SBSB 08 with 350 bp \& 625 bp respectively, P.ostreatus by SBSB 11 with (375 bp), H.ulmarious by SBSA 14, SBSB 13 with 525 bp \& 600 bp respectively (Table 1). RAPD markers revealed genetic diversity among the Pleurotus species with genetic similarity ranging from 0.22 to 0.98 (Table 2 and 3 ).

In UPGMA dendogram (Fig. 1), there were 5 clusters which made several group and sub group. Cluster A with strains namely P.sajor caju \& $P$. citripileatus. Cluster B distinguished a separate species $P$. djamor. Cluster D \& E made from cluster C. $P$. flabellatus separate from cluster E. Cluster D sub grouped with $P$. ostreatus \& $H$. ulmarious. Cluster $\mathrm{F}$ grouped into two sub group, first sub group with $P$. fossulatus $\& P$. florida, second sub group with $P$. platypus \& P. sapidus.

\section{References}

Gokulpalan C, Nair MC.1990. Cultivation of Mushrooms. Mushrooms Tech. Bull. Edited by M.C. Nair, Deptt. of Plant Pathol. College of Agriculture,
Vellayani, Kerala.

Gunde-Cimerman N. 1999. Medicinal Value of the Genus Pleurotus (Fr.) P. Karst. (Agaricales s.l., Basidiomycets). The International Journal of Medicinal Mushrooms. 1: 69-80.

Guzman G.2000. Genus Pleurotus (Jacq. Fr.) $P \quad$ Kumm. (Agaricomycetidenae): Diversity, Taxonomy Problems and Cultural and Traditional Medicinal Uses. The International Journal of Medicinal Mushrooms, 2: 95-123.

Rohlf FJ.1997. NTSYS pc: Numerical Taxonomy and Multivariate analysis system version 2.02h. Exeter software. New York.

Sneath PHA, Sokal RR.1973. Numerical Taxnmy. W.H. Freeman, San Francisco, California.

Stajic M, Milenkovic I, Brceski I, Vukojevic J. Duletic-Lausevic S. 2002. Mycelial growth of and medicinal oyster mushroom [Pleurotus ostreatus(Jaq: Fr.) Kumm.] on seleniumenriched media. International Journal of Medicinal Mushroom, 4: 241-244.

Thayumanavan B, Manikam A.1980. Protein quality of the sporophaore of the fungus Pleurotus sajor caju (fr.) Singer. Indian J. Nutri. Diet., 17: 140-142.

Zervakis GI, Moncalvo JM, Vilgalyas R.2004.Molecular Phylogemy, biogeography and speciation of mushroom species Pleurotus cystidiosus and allied taxa. Microbiology, 150(3):715-726.

\section{How to cite this article:}

Ravi Prakash Mishra, Manjul Pandey, U.K. Tripathi and Singh, M. 2019. Genetic Diversity within the Genus Pleurotus Determined by RAPD Analysis. Int.J.Curr.Microbiol.App.Sci. 8(01): 2870-2875. doi: https://doi.org/10.20546/ijcmas.2019.801.302 